\title{
VISCOUS WRINKLING OF NON-UNIFORM SHEETS
}

Oliver McRae, Alexandros Oratis, James Bird

Department of Mechanical Engineering, Boston University, Boston MA, USA

A centimeter-sized bubble resting at the surface of an ultra-viscous liquid can adopt radial wrinkles as it collapses over a period of seconds. The wrinkling pattern is strikingly similar to those found in elastic sheets. Wrinkles are typically formed on elastic sheets of constant thickness. Yet, the appearance of unevenly spaced interference fringes highlights that the viscous sheet is non-uniform as it wrinkles. Measuring how the thickness varies along the film can allow us to better understand if and how this non-uniformity influences the number and location of the wrinkles.

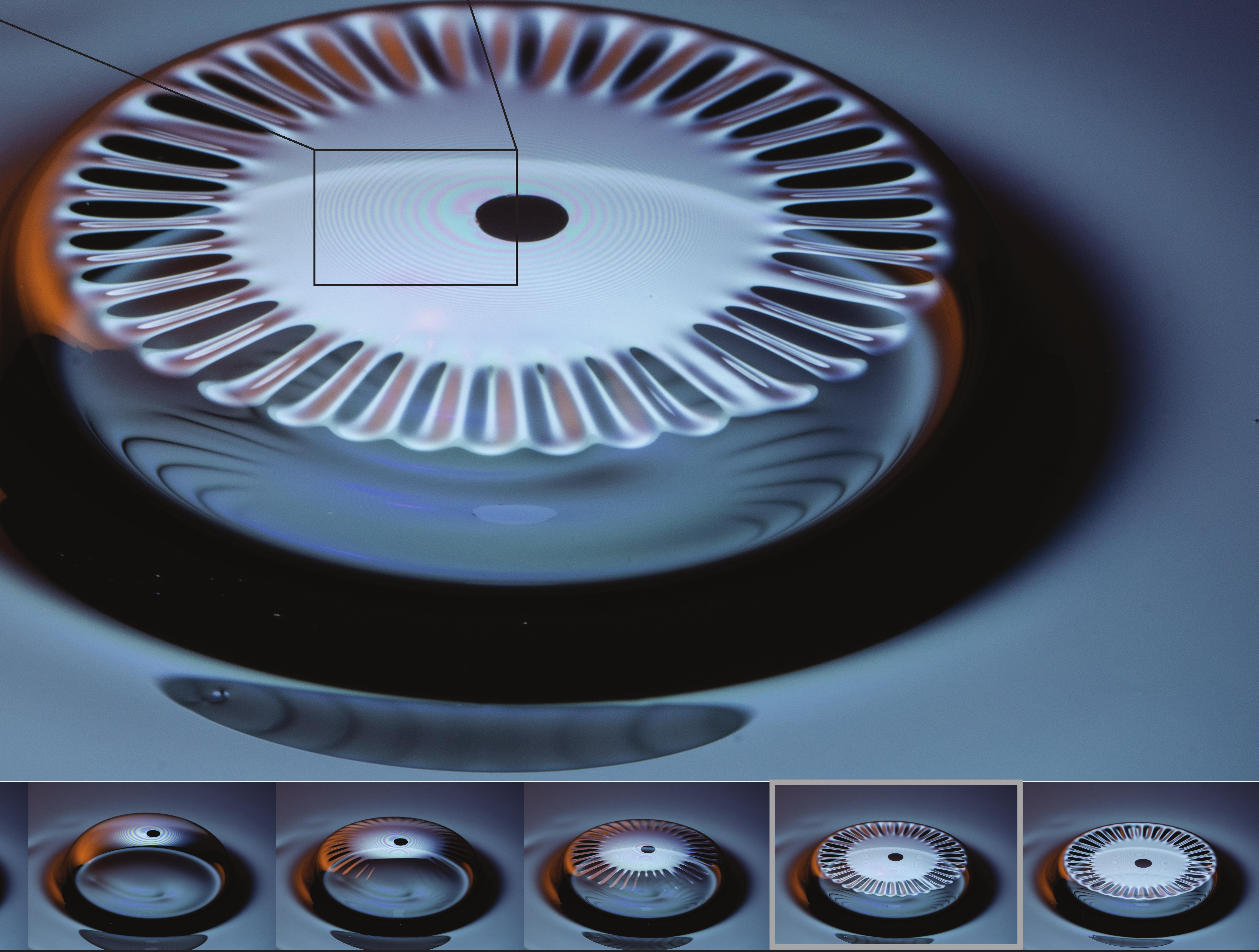

